\begin{tabular}{|c|c|}
\hline Title & A ctive implantable medical device EMI assessment for wireless power transfer operating in LF and HF bands \\
\hline Author(s) & Hikage, T akashi; Nojima, Toshio; Fujimoto, Hiroshi \\
\hline Citation & $\begin{array}{l}\text { Physics in medicine and biology, 61(12), 4522-4536 } \\
\text { https://doi.org/10.1088/0031-9155/61/12/4522 }\end{array}$ \\
\hline Issue Date & 2016-06-22 \\
\hline Doc URL & http:/hdl.handle.net/2115/66209 \\
\hline Rights & $\begin{array}{l}\text { This is an author-created, un-copyedited version of an article published in Physics in Medicine \& Biology. IOP } \\
\text { Publishing Ltd is not responsible for any errors or omissions in this version of the manuscript or any version derived } \\
\text { from it. The V ersion of Record is available online at } 10.1088 / 0031-9155 / 61 / 12 / 4522 \text {. }\end{array}$ \\
\hline Type & article (author version) \\
\hline File Information & manuscript.pdf \\
\hline
\end{tabular}

Instructions for use 


\title{
Active Implantable Medical Device EMI Assessment for Wireless Power Transfer Operating in LF and HF Bands
}

\author{
Takashi Hikage $^{1}$, Toshio Nojima ${ }^{1}$ and Hiroshi Fujimoto ${ }^{2}$ \\ ${ }^{1}$ Graduate School of Information Science and Technology, Hokkaido University \\ Kita14, Nishi9, Kita-ku, Sapporo, Hokkaido, Japan \\ ${ }^{2}$ Medtronic Japan Co., Ltd. Tokyo Japan \\ E-mail: hikage@wtemc.ist.hokudai.ac.jp
}

\begin{abstract}
The electromagnetic interference (EMI) imposed on active implantable medical devices by wireless power transfer systems (WPTSs) is discussed based upon results of in vitro experiments. The purpose of this study is to present comprehensive EMI test results gathered from implantable-cardiac pacemakers and implantable cardioverter defibrillators (ICDs) exposed to the electromagnetic field generated by several WPTSs operating in low-frequency $(70 \mathrm{kHz}-460 \mathrm{kHz})$ and high-frequency $(6.78 \mathrm{MHz})$ bands. The constructed in vitro experimental test system based upon an Irnich's flat torso phantom was applied. EMI test experiments are conducted on 14 types of WPTSs including Qi-compliant system and EV-charging WPT system mounted on current production EVs. In addition, a numerical simulation model for Active Implantable Medical Device (AIMD) EMI estimation based on the experimental test system is newly proposed. The experimental results demonstrate the risk of WPTSs emitting intermittent signal to affect the correct behavior of AIMDs when operating at very short distances. The proposed numerical simulation model is applicable to obtain basically the EMI characteristics of various types of WPTSs.
\end{abstract}

Keywords: active implantable medical device, electromagnetic interference, wireless power transfer, Qi-compliant, finite element method 


\section{Introduction}

The electromagnetic fields emitted/leaked from wireless devices raise the concern that they may cause other electronic devices to malfunction. Accordingly, investigations of electromagnetic interference (EMI) are now critical. EMI effects on medical equipment, including active implantable medical devices (AIMD), such as implantable-cardiac pacemakers and -cardioverter-defibrillators (ICD), should be investigated because these are used by widely scattered members of the general public. In particular, the EMI caused by mobile phone systems is being investigated on a massive scale (Barbaro et al 1996, Irnich et al 1996, Toyoshima et al 1996, Hayes et al 1997, Tang et al 2005). In order to reduce the EMI risk pose to AIMDs, some guidelines on EMI suppression define a minimum safe distance in Guidelines on the use of radio communication equipment such as cellular telephones - Safeguards for electric medical equipment (1997), ANSI/AAMI PC69 (1999), Guidelines on the use of radio communications equipment for implanted medical devices (2005) and so on.

Recently, wireless power transfer (WPT) technologies using resonant coupling have been attracting attention (Kurs et al 2007). The technologies are expected to achieve wireless charging and power supply functions for low-power and high power applications such as home appliances, electric vehicles (EV), and other electric systems. Since these systems can generate reactive fields of high field strength, it is important to assess exposure level for human safety. The International Commission on Non-ionizing Radiation Protection (ICNIRP) (ICNIRP 1998, 2010) and IEEE International Committee on Electromagnetic Safety (ICES) Technical Committee 95 (IEEE 2002, 2006) have issued safety guidelines/standards for human protection from electromagnetic fields. Investigations of compliance with the basic restrictions defined in SAR, induced currents and electric fields have been well conducted (Hirata et al 2013, Christ et al 2012, Sunohara et al 2015). However, there is another concern that, when an implantable medical device patient is close to WPT system (WPTS), the electromagnetic fields may be strong enough to affect pacemaker operation. The threshold for EMI occurrence on AIMD is often well below guidelines/standards levels for human exposure to RF fields. In addition, the electromagnetic field distribution generated by a resonant coupling based WPTS is complicated and varies with the coupling condition (e.g. frequency, air-gap, etc.) (Hikage et al. 2012). Therefore it is important to assess the pacemaker (including ICD) EMI risk posed by a WPTS by means of reliable measurement techniques. However, no rigorous assessment report based on WPTS experiments is known.

This is the first study to describe the EMI characteristics of AIMDs based on assessments of various types of WPTSs including Qi-wireless-charging-compliant system and EV-WPT system (IEC - TC 69). We test these WPTSs to estimate the EMI experienced by more than 25 types of implantable-cardiac pacemakers and ICDs. In addition, a novel numerical WPT / AIMD-EMI estimation methodology based on the experimental test system is proposed. Evaluation of interference voltages on the pacemaker input register exposed to HF band WPT coils demonstrates the applicability of the proposed estimation method. Finally, to validate the simulation result, the calculated interference voltage is compared with the EMI characteristics obtained by in vitro EMI 
experiments.

\section{Method and Materials for EMI experiments}

\subsection{In vitro AIMD EMI test system for wireless power transfer system}

The in vitro EMI test system is developed based upon Irnich's flat torso phantom (Irnich et al 1996). The basic configuration of the measurement system and a picture of the actual system are shown in figures 1 and 2, respectively. The basic configuration mirrors those used for RF device tests such as cellular (Barbaro et al 1996, Irnich et al 1996, Toyoshima et al 1996, Hayes et al 1997, Tang et al 2005), RFID (Seidman et al 2010, Mattei et al 2013) and EAS (Guidelines on the use of radio communications equipment for implanted medical devices in Japan 2005). As shown in figure 1, a simulated ECG signal generator / AIMD monitor supplies the simulated ECG signal to the pacemakers and ICDs through the electrodes and lead wire. Figure 2 gives the dimensions of the human torso phantom. Separation of the simulated ECG signals between the atrium and the ventricle leads was maintained at a level greater than $20 \mathrm{~dB}$, which enables high sensitivity EMI tests to be conducted on dual chamber pacemakers. The ECG signal generator, to which a resistor ( $>2 \mathrm{k} \Omega$ ) was connected, supplied the simulated ECG signal to the AIMD through the electrodes and leads. Pacemakers and ICDs need to sense the simulated ECG signal in order to operate properly. The chart recorder and the oscilloscope recorded the simulated ECG signal and output pulse of the AIMD to estimate the occurrence of EMI.

The AIMDs of 5 manufacturers, which included pacemakers, CRT-P, CRT-D and ICDs, were tested. The devices included both dual-chamber and triple-chamber AIMDs. These devices were provided by the Japan Arrhythmia Device Industry Association. As shown in Table 1, in order to obtain sufficiently conservative EMI test results, the sensitivity and refractory period of the pacemakers were set at maximum (most sensitive) and minimum, respectively. The stimulation mode was programmed to AAI (Atrial Atrial Inhibited) or VVI (Ventricular Ventricular Inhibited). Sensing and pacing polarity were either unipolar mode or bipolar mode. In addition, the pacing rate was set to 60 ppm (pulses per minute).

\subsection{Tested wireless power transfer systems}

14 types of WPTSs including some for Mobile/Portable application (12 devices, $\leq 50 \mathrm{~W}$ ), for EVcharging ( 2 devices) were tested. The operation frequency of the tested WPTs ranged from $70 \mathrm{kHz}$ to 6.78 MHz. The transmission power range was up to $3 \mathrm{~kW}$, which is determined considering the current trend of the standard,. The tested WPTSs for mobile/portable application included some commercially available Qi certification devices.

Figure 3 defines the position of the Mobile/Portable-WPTS relative to the torso phantom in the test. For the Mobile/Portable-WPTS, we tested two different transmitting conditions. One assumed the power transfer mode in which the receiver device (Rx-device) was mounted on the transmitting device (Tx-device). The other was standby mode where the Rx-device was absent (un-mounted). Some of the tested Tx-devices emit intermittent signals in standby mode for Rx-device detection.

Figure 4 shows the basic configuration and definition of the position of the torso phantom relative 
to the EV-WPTS in the tests. 2 types of EV-WPTS mounted on current production EVs were tested. Those systems have different coil structures. A WPTS transfers electrical energy from a power source to an electrical load via electric and/or magnetic fields or waves between a transmitting coil (Tx-coil) and a receiving coil (Rx-coil). The Rx-coil was mounted beneath the rear seat of the EV in both types of the WPTS tested. The systems tested in this study emitted continuous wave (CW) with operation frequency of $85 \mathrm{kHz}$. Typical characteristics of the EV-WPTS tested are summarized in Table 2. Three different practical scenarios (Scenarios (A), (B) and (C)) were designed for conservative EMI assessments. Scenarios (A) and (B) were designed to address the effects of different field distributions and the relative position of the human body to the Tx- and Rx- coils close to the EV. Scenario (C) covered the case wherein a human being is sitting or lying on the rear seat of the EV.

\subsection{Fundamental Test procedure}

In order to assess the EMI of pacemakers and ICDs, we conducted "inhibition tests" and "asynchronous tests." The inhibition test examines the missing of pacing pulses generated by the AIMD. There is no injection of the simulated pulse in inhibition tests. An example of an inhibition test result is shown in figure 5 (a). The required pacing pulse is inhibited due to EMI. The asynchronous test examines the generation of fixed rate asynchronous pulses. The simulated cardiac pulses are injected in asynchronous tests. An example of an asynchronous test result is shown in figure 5 (b). When there is no EMI, the AIMD senses the simulated pulse and the pacing pulse is inhibited. However, an asynchronous pulse is generated when the AIMD suffers noise and switches to noise reversion mode. We also investigated inappropriate tachyarrhythmia detection and the delivery of therapy or shock in the experiments on ICDs. To obtain conservative EMI estimation results, sensitivity and refractory period are maximum (most sensitive) and minimum, respectively. The test procedure for Mobile/Portable-WPT is identical to the one proposed for RFID reader/writers (Guidelines on the use of radio communications equipment for implanted medical devices 2005). On the other hand, we propose a new procedure for EV-WPTS as shown in figure 6 , to suit the scenarios (Hikage et al 2015) . The common points for the tests are as follows:

1) First, program the sensitivity and refractory period of pacemakers and ICDs to the maximum sensitivity and the minimum time, respectively.

2) Record the ECG signal for each mode. The distance between the body of the EV and the human torso phantom front surface is decreased when no interference occurs. In this case, the maximum interference distance (distance at which EMI disappears) is determined and recorded in centimeters.

3) Step down the sensitivity of pacemakers and ICDs in five levels (maximum, $1.0 \mathrm{mV}, 2.4 \mathrm{mV}$, $5.6 \mathrm{mV}$, and minimum) and record the maximum interference distance.

4) Carry out experiments for all combinations of the scenario and AIMDs. The operating modes of pacemakers and ICDs include unipolar and bipolar mode, AAI and VVI mode.

Overviews of scenario (A) and (C) are shown in figures 7 and 8, respectively.

\section{EMI Test Results}

The in vitro EMI experiments were conducted using the test system, with the procedure and the 
assumed scenario described above. The measured results are summarized in Table 3. For 5 devices of mobile/portable-WPT, reactions were observed in pacemaker or ICD tests. All observed EMI events occurred in standby mode (The tested Tx-device emitted an intermittent signal). The maximum interference distances ranged from 1 to $2 \mathrm{~cm}$. The reactions observed included pacing inhibition and inappropriate pacing (The max. reaction level; pacemaker: level 2, ICD: Level 1 (Guidelines on the use of radio communications equipment for implanted medical devices 2005). All EMI events observed were transient responses. Once the RF source was turned off, the PMs/ICDs returned to normal operation within few seconds, and no permanent variation in the programmed parameters occurred.

For EV-WPTSs, each scenario tested was verified to yield normal operation during EMI testing. No inappropriate reaction, such as missing of pacing pulses, generation of asynchronous pulses for pacemaker functions and inappropriate tachyarrhythmia detection and delivery of therapy for ICDs was observed under EMI testing.

\section{Numerical estimation of AIMD-EMI due to WPT}

A novel numerical WPT/AIMD-EMI estimation methodology based upon finite element method (FEM) analysis is presented. This assessment methodology can be applied to both Mobile/PortableWPTS and EV-WPTS. Here, an example for HF-band magnetic resonance type WPTS proposed by a Kurs et al (2007) is introduced. It assumes that WPT coils operating in the HF band or below might cause EMI on the AIMDs through the interference voltage induced by the magnetic flux that is interlinked with the one turn coil formed with the AIMD, the lead-wire and the direct body current path in the torso phantom (ISO/IEC 2011 TR 20017). Therefore, fundamental EMI characteristics obtained here can demonstrate the applicability of the proposed numerical estimation method for both LF and HF bands WPT systems. The FEM analysis was carried out using the commercial software EMPro (EMPro 2013).

\subsection{Numerical model of torso phantom including pacemaker and leads}

Based on the experimental torso phantom, we constructed a numerical EMI estimation model consisting of a torso phantom and virtual pacemaker. The torso phantom model for numerical estimation, which contains the pacemaker model, is shown in figure 9. This numerical phantom is composed of an acrylic tank and uses the same saline solution as the experimental phantom. As shown in the figure, the two lead wires (atrial lead and ventricular lead) are connected to the pacemaker's input registers. Most of the AIMDs have two operating modes depending on the configuration of the electrodes. One mode is called "unipolar". In this mode, the AIMD uses its metal housing as the indifferent electrode and the tip electrode at the end of the lead wire as the different electrode. The other is called "bipolar". In this mode, the difference electrode is the tip electrode and the indifferent electrode is a ring electrode, which is located about $1 \mathrm{~cm}$ away from the end of the lead wire. In this paper, we assumed unipolar leads. Actually, the pacing leads configuration plays a key role in the calculation of the EMI effects. However, the configuration is very complex and is not generally made known by pacemaker manufacturers in any detail. In this paper, we used the modeling of a unipolar 
pacemaker leads as simple solid wires. In addition, the interference voltage is evaluated at the register (1 M ohm) on each terminal (Hikage et al. 2012). Therefore, we cannot obtain absolute values of the induced voltages on real pacemaker by the proposed method. This is the limitation of the study using computational approach in a current situation. The dielectric constants and electric conductivities of each material used in the phantom model are summarized in Table 4.

\subsection{HF-band Wireless Power Transfer Coils}

Numerical models of the coils are constructed assuming a WPTS with magnetically-coupled coils as described by Kurs et al (2007). The coil parameters (Suzuki et al. 2013) are summarized in Table 5. In the model, one-loop coils for feeding and receiving power are placed close to the resonant coils, the spacing between one-loop coil and resonant coil is $13 \mathrm{~cm}$. When the distance between the Tx and Rx coils is $1 \mathrm{~m}$, the resonance of the coils occurs close to $10 \mathrm{MHz}$ and the resonant frequency splits into two peaks (lower resonant frequency : $f m$, higher resonant frequency: $f e$ ) under strong coupled resonance conditions. The two resonant modes create different field distributions around the coils.

\subsection{Numerically evaluated interference voltage}

The modeled WPTS coils and the human torso phantom were combined to obtain the interference voltage. Here, coil distance $D$ was $1.0 \mathrm{~m}$, and two different scenarios (scenario-A and scenario-B) are assumed as shown in figure 10. One is when the human torso phantom is located between the Tx- and RX- coils (Figure 10 (a)) and the other is when the phantom located behind the Tx coil (Figure 10 (b)). The interference voltage at the resistor was evaluated by the FEM analysis. The calculation parameters for FEM simulations are summarized in Table 6 . We obtained interference voltages at the pacemaker connectors as a function of the distance $(L)$ between the Tx-coil and the torso phantom. Figure 11 plots the calculated interference voltages at the terminal versus distance $L$ from the surface of Tx-coil, (normalized by maximum value obtained these simulation data), for the cases of figure 10 (a) and (b). Tx-coil input powers were the same for both scenarios. When distance $\mathrm{L}$ is $0 \mathrm{~cm}$, the induced voltage for scenario (a) is more than twice that of scenario (b), even though its input power is the same. This is because the difference in the magnetic field distribution strongly affects the induced voltage. By using FEM simulation to evaluate the interference voltage, accurate estimations can be achieved.

\subsection{Validation study based on AIMD EMI test}

In order to estimate the validity of this simulation method, some AIMD EMI tests for the HF-band WPTS coils were carried out using the same test system described above. The WPTS coils had the same dimensions as the numerical models. Figure 12 shows fabricated WPTS coils and the configuration of the EMI test system. The WPTS coils consisted of single turn coils and resonant coils those were made of copper wire (diameter: $2 \mathrm{~mm}$ ). The input power to the Tx-coil was set to be $10 \mathrm{~W}$ at $10.3 \mathrm{MHz}$, and the Rx-coil was terminated by a $50 \mathrm{Ohm}$ matching load.

The in vitro EMI experiments were conducted using the test system for the scenarios (a) and (b) 
shown in figure 10. Only in scenario (a) were reactions observed for some pacemakers and ICDs. The maximum interference distance $(\mathrm{L})$ was $12 \mathrm{~cm}$. The reactions observed included pacing inhibition and inappropriate pacing. All EMI events observed were transient responses following the results shown in Section 3. In scenario (b), no inappropriate reaction was observed even if distance $L$ was set at 0 $\mathrm{cm}$. From the estimation results shown in figure 11, we find that the interference voltage for scenario (a) at distance $\mathrm{L}$ of $11 \mathrm{~cm}$ is still higher than the value for scenario (b). This means that the calculated interference voltages well predict the basically EMI characteristics obtained by in vitro EMI experiments.

\section{Discussion and concluding remarks}

Typical examples of WPTSs, including mobile/portable-WPTS and EV-WPTS, were tested to estimate the EMI levels and the interference distance for various types of AIMDs available in the Japanese market. This is the first report to detail the AIMD EMI imposed by various types of WPTSs. Our experiments confirmed that such EMI demonstrates a reversible and transient response. From the entire set of measurement results, the characteristics common to all types of WPTSs are summarized as follows;

The observed EMI for pacemakers is either a missing pulse(s) or the undesirable generation of asynchronous pulses. The duration of the EMI occurrences vary from a single pulse to the complete inhibition or continuous pulse generation during WPT emission. The EMI (including whether or not they occur) depends upon the combination of devices and operation mode. In the basic EMI mechanism, the EMF penetration components are detected by the nonlinear responses of the internal circuits of the AIMD (envelope detection) and when the detected signal is similar to any of the targeted physiological signals (frequency of a few $\mathrm{Hz}$ to several hundred $\mathrm{Hz}$ ) and exceeds the threshold, AIMD malfunctions can occur. For the EV-WPTS and AIMD combinations examined, no inappropriate generation of asynchronous pulses for pacemaker functions or inappropriate tachyarrhythmia detection and delivery of therapy for ICDs were observed.

Since the transfer signal of the tested EV-WPTS tested was a continuous wave, no AIMD malfunctions occurred even when the transfer power level of the WPTS was $3 \mathrm{~kW}$ class. If the AIMD are exposed to changing fields such as pulse modulated waves or changing magnetic fields, in particular when pulsed signals with a repetition time close to the physiological heart rhythm, the probability of the EMI occurrence becomes significant.

In addition, a novel numerical WPT/AIMD-EMI estimation methodology based upon FEM analysis was proposed. The developed numerical simulation model can well predict the EMI characteristics created by WPTSs. This assessment methodology can be applied to both Mobile/Portable-WPTSs and EV-WPTSs.

\section{Acknowledgment}

The authors would like to thank the members of Japan Arrhythmia Device Industry Association (JADIA) and Broadband Wireless Forum (BWF) of Japan for their cooperation and support. This 
work was partially supported by Grant-in-Aid for Japan Society for the Promotion of Science (JSPS)

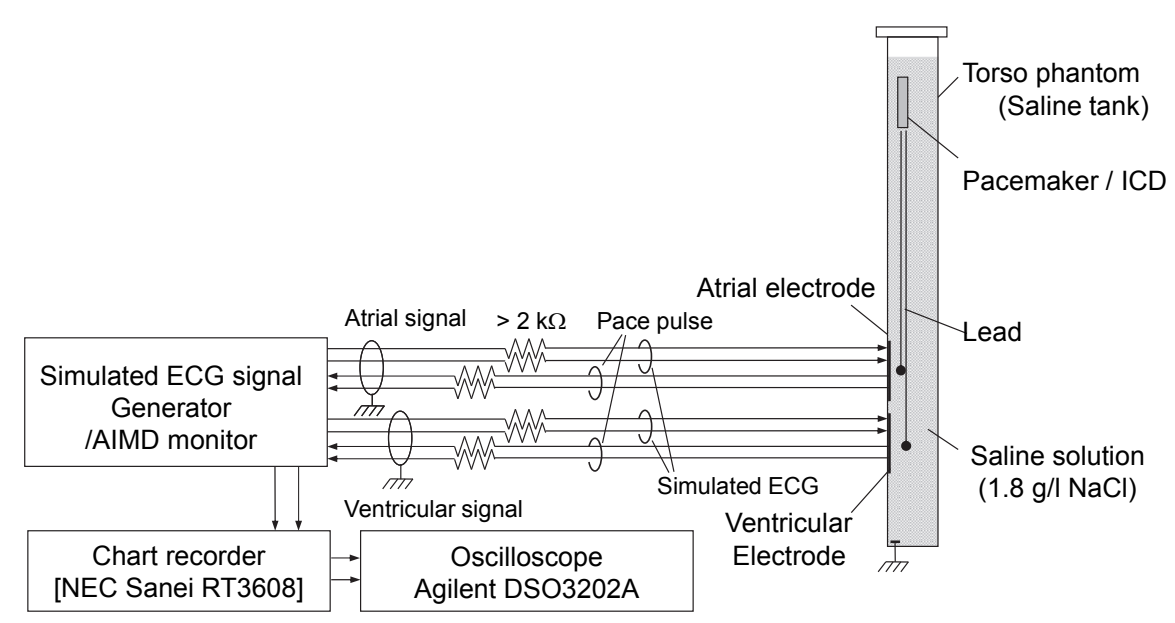

Figure 1. Basic configuration of EMI test phantom.

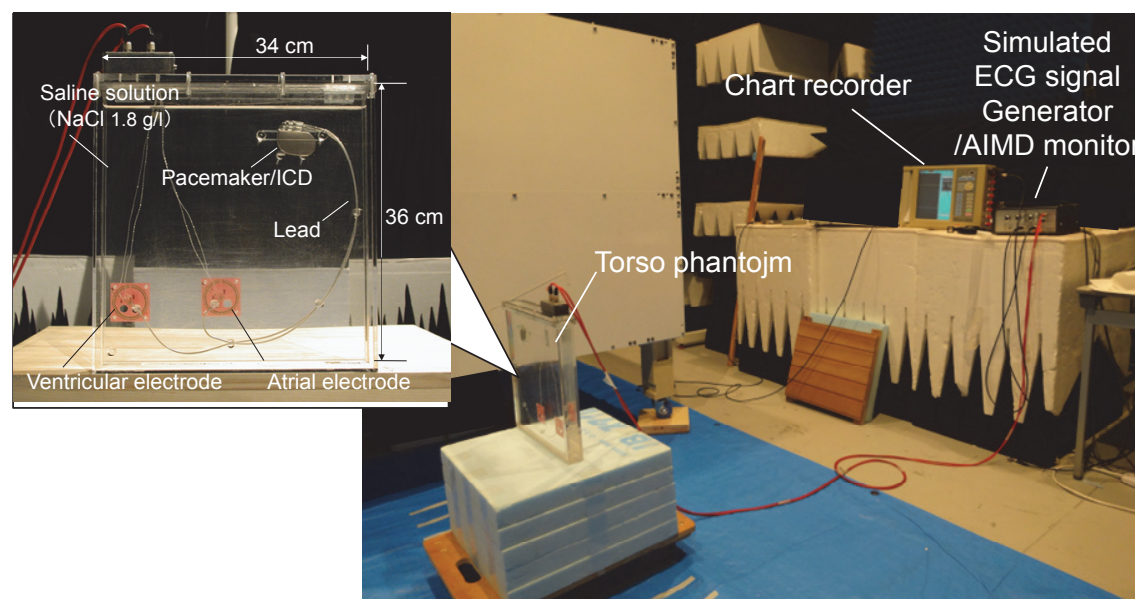

Figure 2. Overview of EMI measurement system.
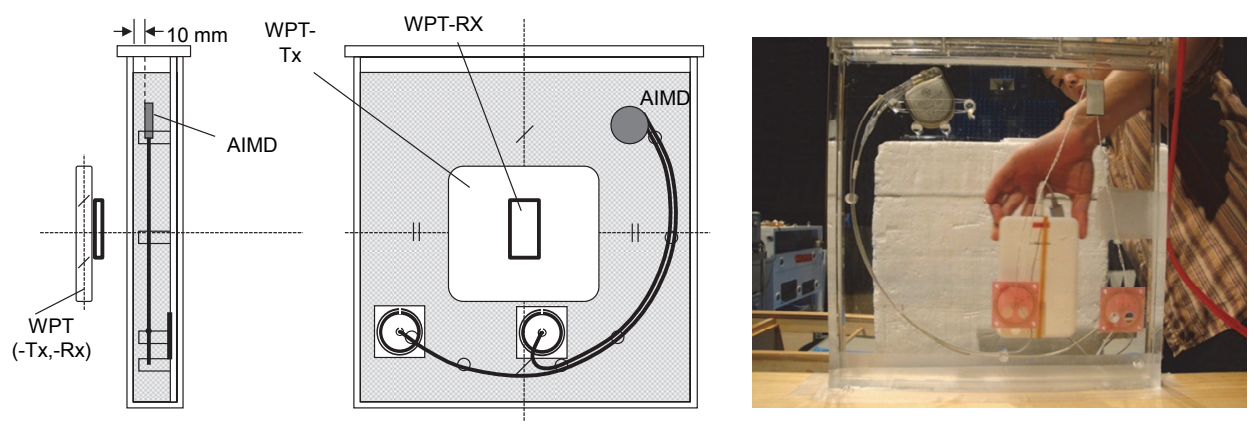

Figure 3. Definition of the position of the mobile/portable-WPTS relative to torso phantom in AIMD-EMI test. 

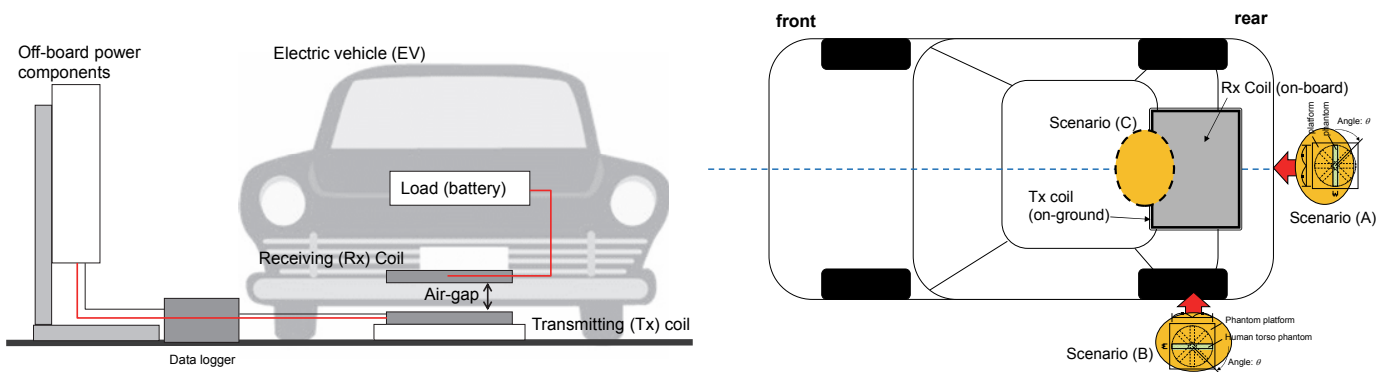

Figure 4. Basic configuration of EV-WPTS and definition of the position of torso phantom in AIMD-EMI test. 3

4

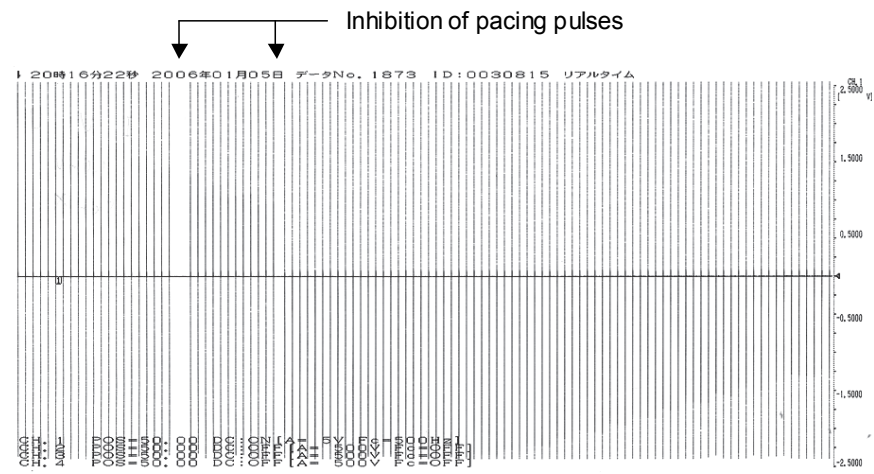

(a)

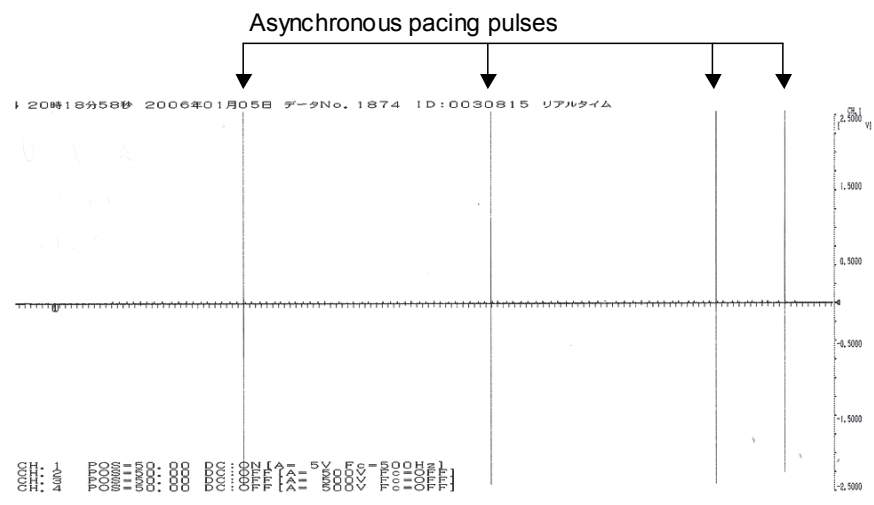

(b)

Figure 5. An example of (a) inhibition and (b) asynchronous test results. 


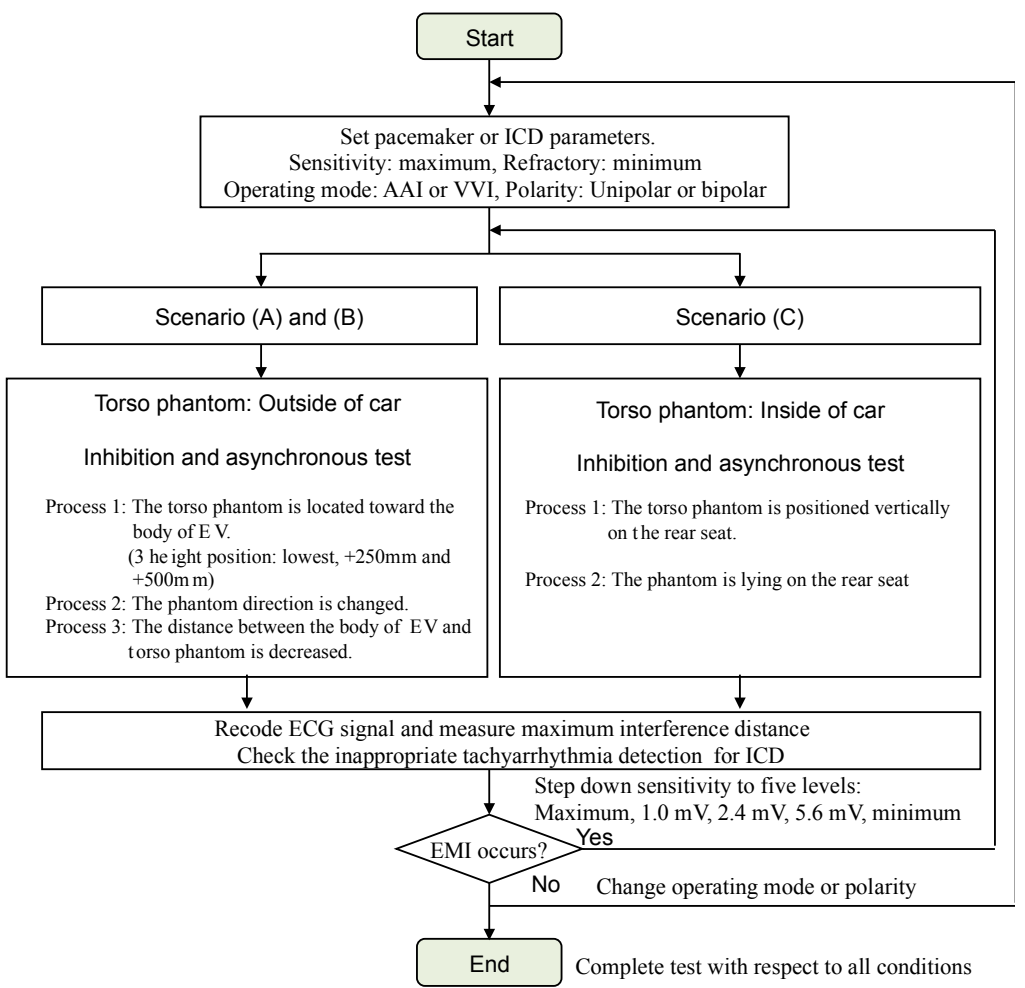

Figure 6. Test procedure for EV-WPTS.
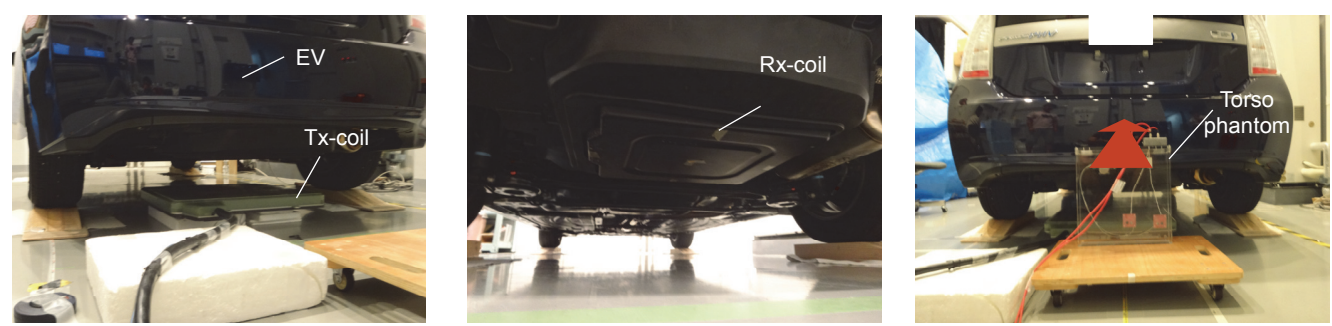

Figure 7. An example of test under scenario (A).

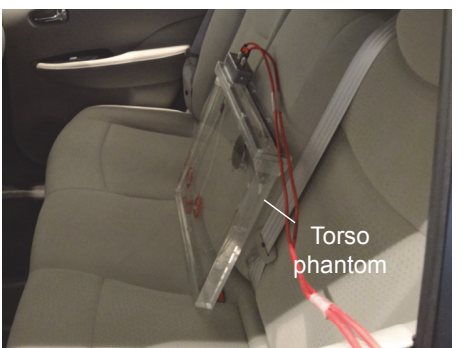

Sitting

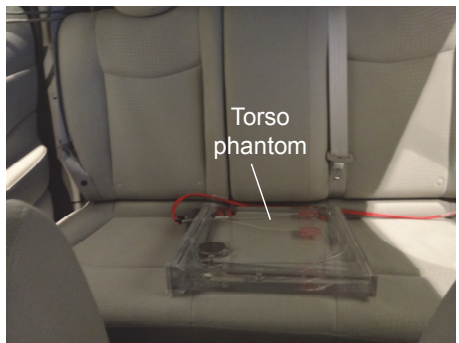

Lying

Figure 8.An example of test under scenario (C). 

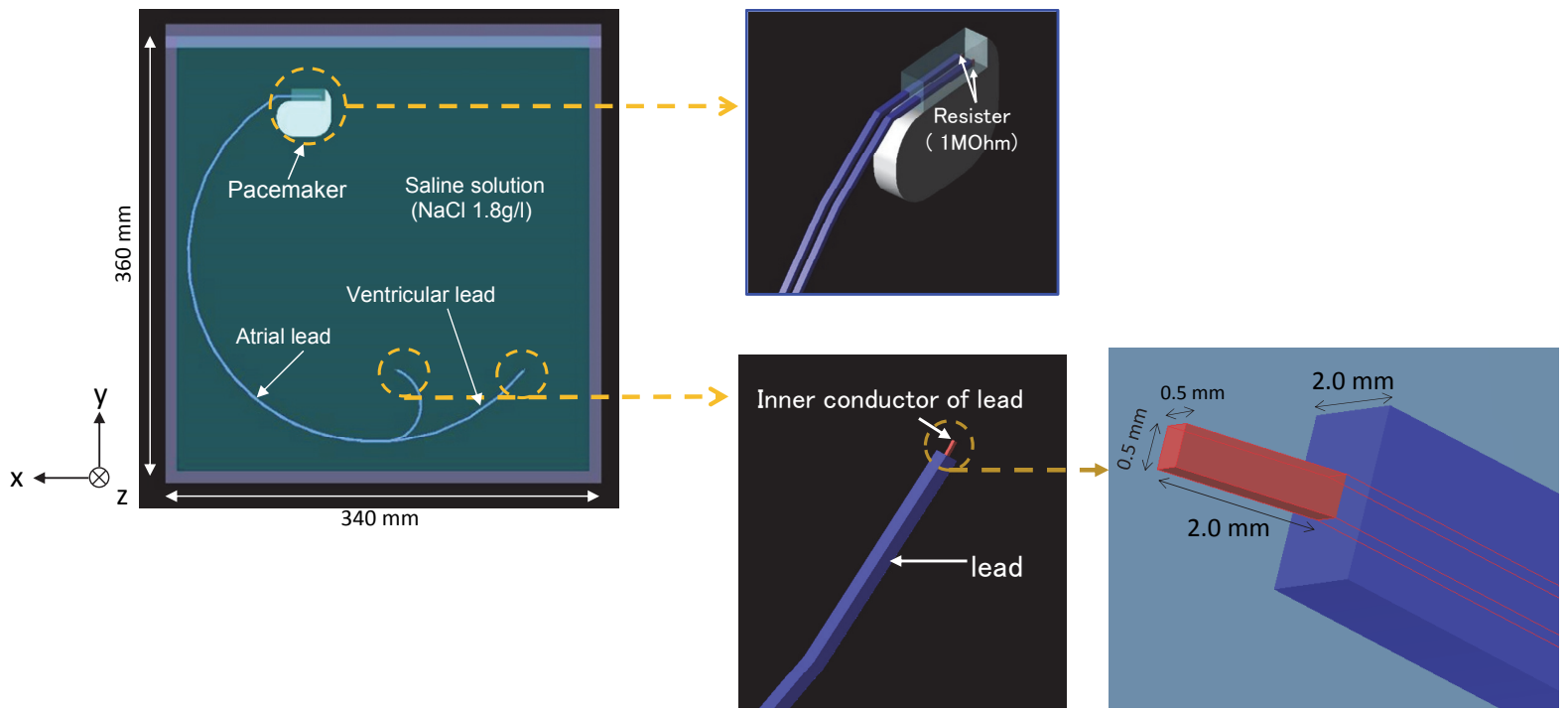

Figure 9. Numerical model of torso phantom for pacemaker EMI estimation.
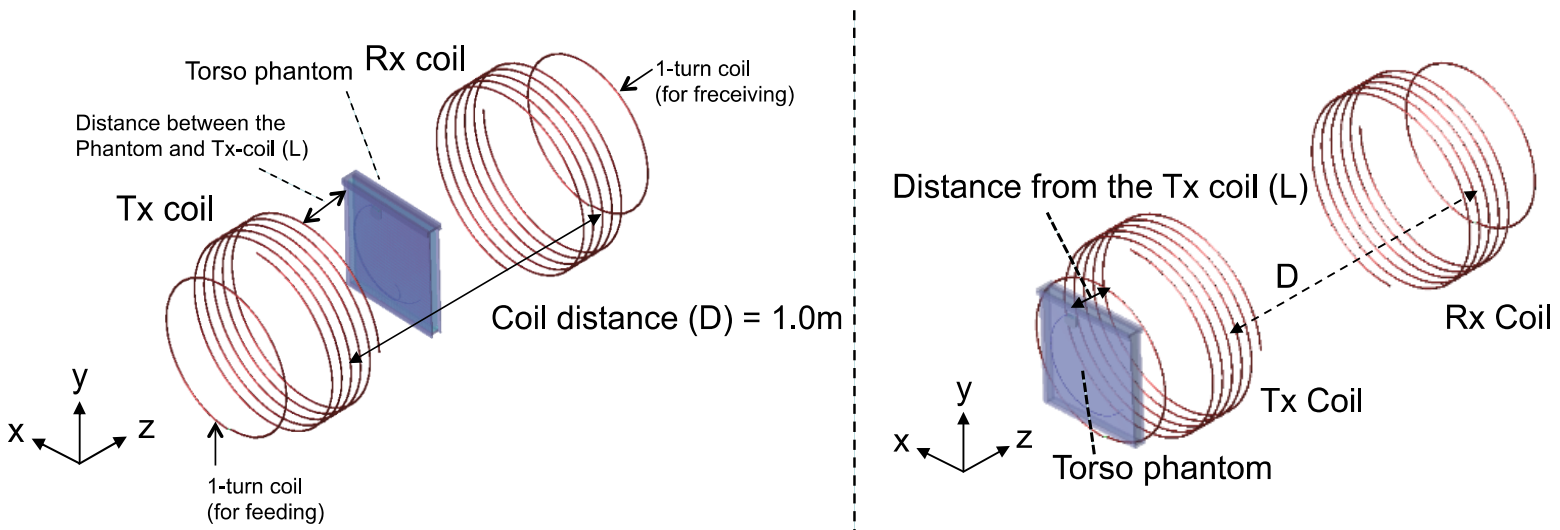

7

scenario-(a)

scenario-(b)

Figure 10. HF-band wireless power transfer coils and human torso phantom. 


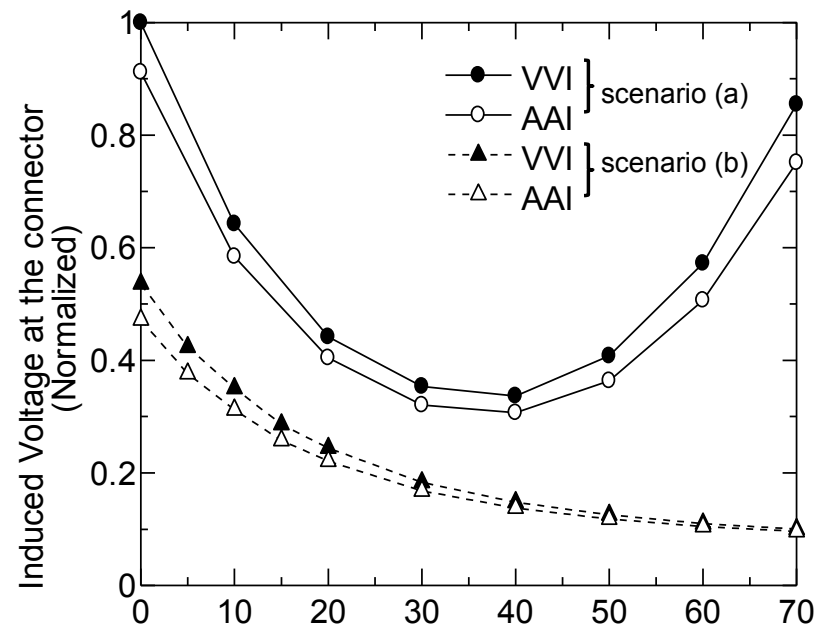

Distance from the transmission coil $(\mathrm{L})[\mathrm{cm}]$

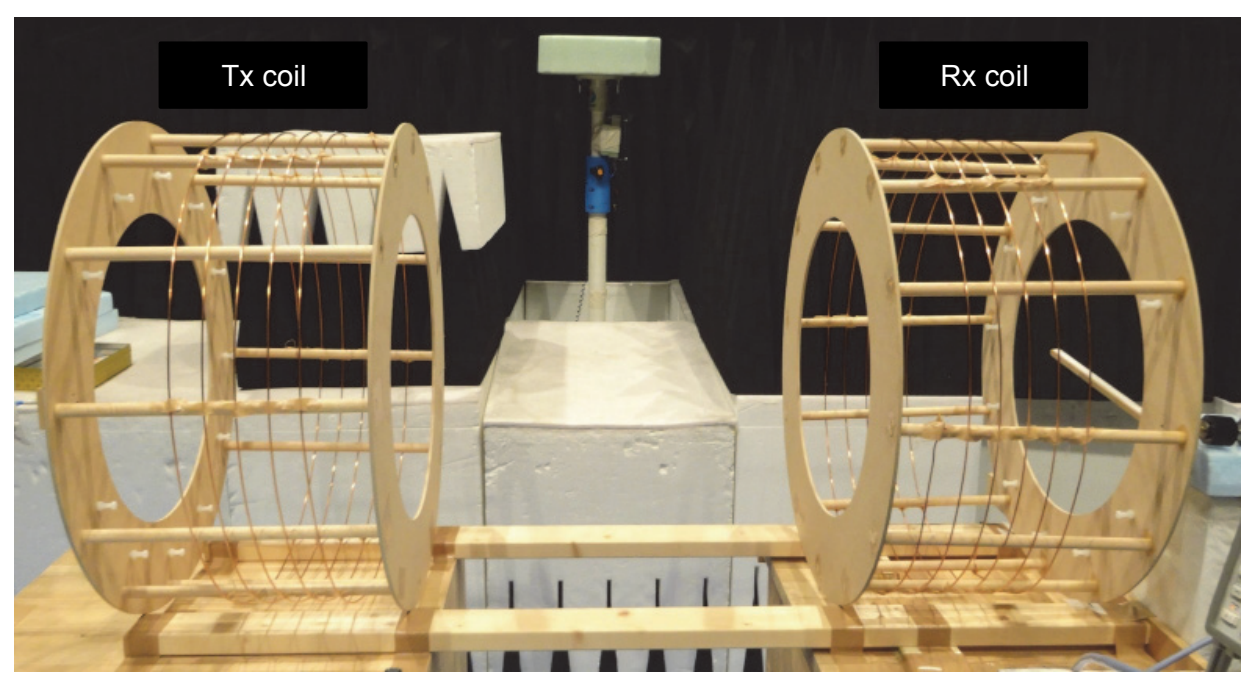

(a) 


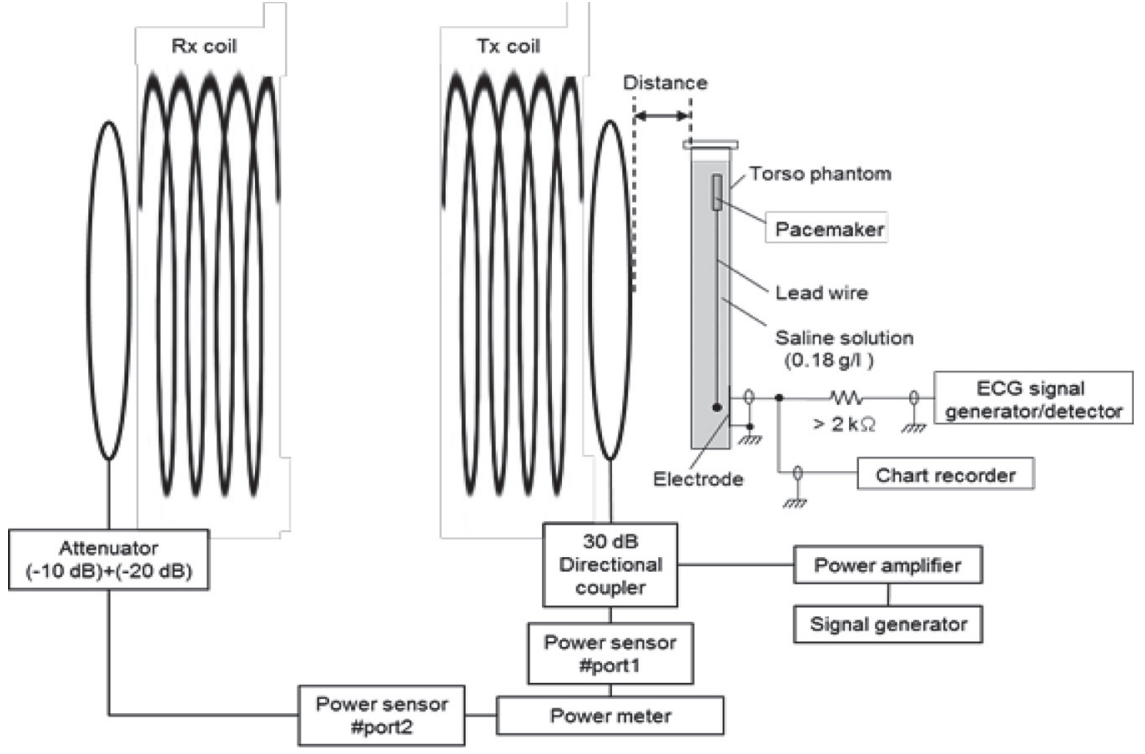

(b)

Figure 12. AIMD EMI Test system for HF-band wireless power transfer coils: (a) WPT coils and (b) configuration of the test system.

5

6

Table 1. Initial Settings of tested AIMDs.

\begin{tabular}{c|c}
\hline Parameter & Value \\
\hline Stimulation mode & AAI or VVI \\
\hline Heart rate & $60 \mathrm{ppm}$ \\
\hline Pacing and sensing polarity & Unipolar or bipolar \\
\hline Pulse amplitude and duration & $\begin{array}{c}\text { Nominal values } \\
\text { (Approximately } 3.5 \mathrm{~V} \text { and } 0.4 \mathrm{~ms} \text { ) }\end{array}$ \\
\hline Sensitivity & Maximum (most sensitive) \\
\hline Refectory period & Minimum \\
\hline
\end{tabular}

12

13

Table 2. Parameters of tested WPTSs for electric vehicles.

\begin{tabular}{|c|c|c|c|c|c|}
\hline & \multicolumn{2}{|c|}{ Wireless power transfer system (WPTS) } & \multicolumn{2}{c|}{ Electric vehicle (EV) } \\
\cline { 2 - 6 } & Frequency & $\begin{array}{c}\text { Transfer power } \\
\text { level }\end{array}$ & $\begin{array}{c}\text { Air-gap } \\
\text { (Distance between } \\
\text { Tx-and Rx coils) }\end{array}$ & Class & Body type \\
\hline Type A & $85 \mathrm{kHz}$ & $2 \mathrm{~kW}$ & $150 \mathrm{~mm}$ & Mid-size car & $\begin{array}{c}5 \text {-door } \\
\text { hatchback }\end{array}$ \\
\hline Type B & $85 \mathrm{kHz}$ & $3 \mathrm{~kW}$ & $165 \mathrm{~mm}$ & $\begin{array}{c}\text { Compact } \\
\text { car }\end{array}$ & $\begin{array}{c}5 \text {-door } \\
\text { hatchback }\end{array}$ \\
\hline
\end{tabular}


Table 3. AIMD-EMI Tested Results for WPT systems.

\begin{tabular}{|c|c|c|c|c|}
\hline \multicolumn{2}{|l|}{ WPT device } & Frequency & Trans. Power & AIMD-EMI Test result \\
\hline \multirow{12}{*}{ Mobile/Portable } & A & $70 \mathrm{kHz}$ & $0.2 \mathrm{~W}$ & \multirow{12}{*}{$\begin{array}{l}\text { - Observed electromagnetic } \\
\text { interference (EMI) at } \\
\text { maximum distance of } \\
\text { Pacemaker: } \leqq 2 \mathrm{~cm} \\
\text { ICD: } \leqq 1 \mathrm{~cm} \\
\text { - Reaction level } \\
\text { Pacemaker : } 2 \\
\text { ICD: } 1 \\
\text { (pacing inhibition and } \\
\text { inappropriate pacing, All EMI } \\
\text { events observed were transient } \\
\text { responses) }\end{array}$} \\
\hline & $\mathrm{B}$ & $100 \mathrm{kHz} \sim 200 \mathrm{kHz}$ & $5 \mathrm{~W}(\max )$ & \\
\hline & $\mathrm{C}$ & $100 \mathrm{kHz} \sim 200 \mathrm{kHz}$ & $5 \mathrm{~W}(\max )$ & \\
\hline & $\mathrm{D}$ & $100 \mathrm{kHz} \sim 200 \mathrm{kHz}$ & $5 \mathrm{~W} \times 2$ & \\
\hline & $E$ & $100 \mathrm{kHz} \sim 200 \mathrm{kHz}$ & $5 \mathrm{~W}$ & \\
\hline & $\mathrm{F}$ & $110 \mathrm{kHz} \sim 183 \mathrm{kHz}$ & $5 \sim 15 \mathrm{~W}$ & \\
\hline & G & $110 \mathrm{kHz} \sim 210 \mathrm{kHz}$ & $1 \sim 15 \mathrm{~W}$ & \\
\hline & $\mathrm{H}$ & $134.5 \mathrm{kHz}$ & $\fallingdotseq 1.4 \mathrm{~W}$ & \\
\hline & 1 & $200 \mathrm{kHz}$ & $13 \mathrm{~W}$ & \\
\hline & $\mathrm{J}$ & $400 \mathrm{kHz}$ & $\fallingdotseq 0.4 \mathrm{~W}$ & \\
\hline & $\mathrm{K}$ & $460 \mathrm{kHz}$ & $40 W(\max )$ & \\
\hline & $\mathrm{L}$ & $6.78 \mathrm{MHz}$ & $18.2 \mathrm{~W}$ & \\
\hline \multirow{2}{*}{ EV charging } & $\mathrm{M}$ & $85 \mathrm{kHz}$ & $2 \sim 3 \mathrm{~kW}$ & \multirow{2}{*}{ No reaction occurred } \\
\hline & $\mathrm{N}$ & $85 \mathrm{kHz}$ & $3 \mathrm{~kW}$ & \\
\hline
\end{tabular}

Blue : Qi certification device

Table 4. Dielectric constant and electric conductivity of material used in phantom model.

\begin{tabular}{|c|c|c|}
\hline & $\varepsilon$ & $\sigma_{r}(\mathrm{~S} / \mathrm{m})$ \\
\hline $\begin{array}{c}\text { Can of Pacemaker, } \\
\text { Lead wire }\end{array}$ & $\begin{array}{c}\text { perfect electric conductor } \\
\text { (PEC) }\end{array}$ \\
\hline Saline solution $(1.8 \mathrm{~g} / \mathrm{l})$ & 86.7 & 0.32 \\
\hline Silicone & 2.7 & 0 \\
\hline Acrylic case & 3 & 0 \\
\hline
\end{tabular}

Table 5. Configurations of wireless power transfer coil.

\begin{tabular}{|c|c|c|}
\hline Number of Turns & \multicolumn{2}{|c|}{5 and $1 / 4$} \\
\hline Pitch & \multicolumn{2}{|c|}{$40 \mathrm{~mm}$} \\
\hline Radius & Input/output loop & $250 \mathrm{~mm}$ \\
\cline { 2 - 3 } & $\begin{array}{c}\text { Transmitting (Tx) Coil } / \\
\text { Receiving (Rx) Coil }\end{array}$ & $300 \mathrm{~mm}$ \\
\hline Diameter of wire & $2 \mathrm{~mm}$ \\
\hline Material & $\begin{array}{c}\text { Copper } \\
\left(\sigma=5.813 \times 10^{7} \mathrm{~S} / \mathrm{m}\right)\end{array}$ \\
\hline $\begin{array}{c}\text { Distance between } \\
\text { Tx and Rx coils } \\
(D)\end{array}$ & $1.0 \mathrm{~m}$ or $1.5 \mathrm{~m}$ \\
\hline
\end{tabular}

Table 6. Simulation parameters.

\begin{tabular}{|c|c|c|}
\hline & FEM Solver & Direct Solver \\
\hline & er of Basis Function & 2 \\
\hline & undary Conditions & 2nd order radiation boundary condition \\
\hline Estim & tion Frequency Range & $8 \sim 13 \mathrm{MHz}$ \\
\hline \multirow{5}{*}{$\begin{array}{l}\text { FEM } \\
\text { Mesh } \\
\text { size }\end{array}$} & Tx and Rx coils & $5 \mathrm{~mm}$ \\
\hline & Cardiac pacemaker & $5 \mathrm{~mm}$ \\
\hline & Torso phantom & $10 \mathrm{~mm}$ \\
\hline & Conductor Edge Mesh & $0.2 \times$ estimation conductor width \\
\hline & Conductor Vertex Mesh & $0.2 \times 0.3 \times$ estimation conduc \\
\hline
\end{tabular}




\section{References}

American National Standards Institute/Association for the Advancement of Medical Instrumentation (ANSI/AAMI) 1999 Active implantable medical devices-electromagnetic compatibility-EMC test protocols for implantable cardiac pacemakers and implantable cardioverter defibrillators ANSI/AAMI PC69: 1999 (Arlington, VA: Association for the Advancement of Medical Instrumentation)

Barbaro V, Bartolini P, Donato A and Militello C 1996 Electromagnetic interference of analog cellular telephone with pacemakers J. Pacing and Clinical Electrophysiology 19 1410-18

Christ A, Douglas M G, Roman J M, Cooper E B, Sample A P, Waters B H, Smith J R and Kuster N 2012 Evaluation of wireless resonant power transfer systems with human electromagnetic exposure limits IEEE Trans. Electromagn. Compat. 55 265-74

Electromagnetic Medical Equipment Study Group 1997 Guidelines on the use of radio communication equipment such as cellular telephones - Safeguards for electric medical equipment proc. the EMC Conf. Japan

EMPro 2013 EM Simulation Software (Keysight Technologies)

Ministry of Internal Affairs and Communication of Japan 2005 Guidelines on the use of radio communications equipment for implanted medical devices

Hayes D L, Wang P J, Reynolds D W, Estes III M, Griffith J L, Steffens R A, Carlo G L, Indlay G K.and Johnson C M 1997 Interference with cardiac pacemakers by cellular telephones New Engl. J. Med. 336 1473-79

Hikage T, Kawamura Y, Nojima T and Cabot E 2012 Numerical Assessment Methodology for Active Implantable Medical Device EMI due to Magnetic Resonance Wireless Power Transmission Antenna Proc. Int. Symp. Electromagnetic Compat. (EMC EUROPE) (IEEE) pp 1-6

Hikage T, Shirafune M, Nojima T and Fujimoto H 2015 In-vitro Assessment of Electromagnetic Interference Due to Electric Vehicle Wireless Power Transfer System on Active Implantable Medical Devices Proc. 9th Int. Symp. Med. Inf. and Comm. Tech. (ISMICT2015) 59-62

Hirata A, Ito F and Laakso I 2013 Confirmation of quasi-static approximation in SAR evaluation for a wireless power transfer system Phys. Med. Biol. 58 N241-N249

ICNIRP 1998 Guidelines for limiting exposure to time-varying electric, magnetic, and electromagnetic fields (up to 300 GHz) Health Phys. 74 494-521

ICNIRP 2010 Guidelines for limiting exposure to time-varying electric and magnetic fields (1 Hz to $100 \mathrm{kHz}) \mathrm{Health}$ Phys. $99818-36$

IEC - $\quad$ TC 69, Electric road vehicles and electric in dustrial trucks, Projects/Publications,http://www.iec.ch/dyn/www/f?p=103:23:0::::FSP_ORG_ID,FSP_LANG_ID:1255,25.

IEEE 2002 IEEE standard for safety levels with respect to human exposure to electromagnetic fields, 0 to $3 \mathrm{kHz}$

IEEE 2006 IEEE standard for safety levels with respect to human exposure to radio frequency electromagnetic fields, $3 \mathrm{kHz}$ to $300 \mathrm{GHz}$ IEEE C95.1

ISO/IEC 2011 Information technology_radio frequency identification for item management-electromagnetic interference impact of ISO/ICE 18000 interrogator emitters on implantable pacemakers and implantable cardioverter defibrillators TR 20017

Irnich W, Batz L, Muller R and Tobisch R 1996 Electromagnetic interference of pacemakers by mobile phones J. Pacing and Clinical Electrophysiology 19 1431-46

Kurs A, Karalis A, Moffatt R, Joannopoulos J D, Fishier P and Soljacic M 2007 Wireless Power Transfer via Strongly Coupled Magnetic Resonances Science 317 83-86

Mattei E Censi F, Delogu A, Ferrara A and Calcagnini G 2013 Setups for in vitro assessment of RFID interference on pacemakers Phys. Med. Biol. 58 5301-16

Seidman S J, Brockman R, Lewis B M, Guag J, Shein M J, Clement W J, Kippola J, Digby D, Barber C and Huntwork D 2010 In vitro tests reveal sample radiofrequency identification readers inducing clinically significant electromagnetic interference to implantable pacemakers and implantable cardioverter-defibrillators Heart Rhythm 7 99-107

Sunohara T, Hirata A, Laakso I, Santis V D and Onishi T 2015 Evaluation of non-uniform field exposures with coupling factors Phys. Med. Biol. 60 8129-40

Suzuki T, Hikage T, Nojima T 2013 Numerical Assessment Method for Implantable Cardiac Pacemaker EMI Triggered by 10MHz-band Wireless Power Transfer Coils Proc. of IEEE MTT-S Int. Micro Workshop WP4-4

Tang C-K, Chan K-H, Fung L-C and Leung S-W 2009 Electromagnetic interference immunity testing of medical equipment to second- and third-generation mobile phones IEEE Trans EMC 51 659-64

Toyoshima T, Tsumura M, Nojima T and Tarusawa Y 1996 Electromagnetic interference of implantable cardiac pacemakers by portable telephones Japanese J. Cardiac Pacing and Electro-physiology 12 488-97 